\title{
Baixa Dose de Bupivacaína Isobárica, Hiperbárica ou Hipobárica para Raquianestesia Unilateral ${ }^{\star}$ Low Dose Isobaric, Hyperbaric, or Hypobaric Bupivacaine for Unilateral Spinal Anesthesia
}

Luiz Eduardo Imbelloni, TSA ${ }^{1}$, Lúcia Beato, TSA², Marildo A Gouveia, TSA ${ }^{3}$, José Antônio Cordeiro ${ }^{4}$

\section{RESUMO}

Imbelloni LE, Beato L, Gouveia MA, Cordeiro JA - Baixa Dose de Bupivacaína Isobárica, Hiperbárica ou Hipobárica para Raquianestesia Unilateral.

JUSTIFICATIVA E OBJETIVOS: A raquianestesia unilateral pode apresentar vantagens principalmente em pacientes em regime ambulatorial. Baixa dose da solução anestésica, velocidade lenta da injeção espinal e a posição lateral facilitam a obtenção da distribuição unilateral na raquianestesia. Foram comparadas soluções isobárica, hiperbárica e hipobárica de bupivacaína com objetivos de se obter raquianestesia unilateral em pacientes submetidos a intervenções cirúrgicas ortopédicas em regime ambulatorial.

MÉTODO: Cento e cinqüenta pacientes foram aleatoriamente separados em três grupos para receberem $5 \mathrm{mg}$ de bupivacaína a 0,5\% isobárica (Grupo Iso), ou 5 mg de bupivacaína a 0,5\% hiperbárica (Grupo Hiper), ou 5 mg de bupivacaína a 0,15\% hipobárica (Grupo Hipo). As soluções foram administradas no interespaço $L_{3}$ $L_{4}$ com o paciente na posição lateral e permanecendo nesta posição por 20 minutos. A anestesia sensitiva foi avaliada pelo teste da picada da agulha. O bloqueio motor avaliado pela escala modificada de Bromage. Ambos os bloqueios foram comparados com o lado não operado e entre si.

RESULTADOS: Existiu diferença significativa entre o lado operado e não-operado em todos os três grupos aos 20 minutos, mas maior freqüência de raquianestesia unilateral foi obtida com as soluções hiperbárica e hipobárica de bupivacaína. Bloqueio sensitivo e motor foram observados em 14 pacientes do Grupo Iso, 38 pacientes no Grupo Hiper e 40 pacientes no Grupo Hipo. Não ocorreram alte-

${ }^{*}$ Recebido do (Received from) Instituto de Anestesia Regional do Hospital de Base da Faculdade de Medicina de São José do Rio Preto (FAMERP), São José do Rio Preto, SP

1. Diretor do Instituto de Anestesia Regional do Hospital de Base da FAMERP Anestesiologista da Casa de Saúde Santa Maria e Clínica São Bernardo, Rio de Janeiro, $R J$

2. Anestesiologista da Casa de Saúde Santa Maria e Clínica São Bernardo, Rio de Janeiro, RJ

3. Diretor do Instituto de Anestesia Regional do Hospital de Base da FAMERP

4. Professor da Faculdade de Medicina São José Rio Preto, SP

Apresentado (Submitted) em 5 de dezembro de 2005

Aceito (Accepted) para publicação em 27 de fevereiro de 2007

Endereço para correspondência (Correspondence to):

Dr. Luiz Eduardo Imbelloni

Av. Epitácio Pessoa, 2356/203 - Lagoa

22471-000 Rio de Janeiro, $R J$

E-mail:dr.imbelloni@terra.com.br

(C) Sociedade Brasileira de Anestesiologia, 2007 rações hemodinâmicas em nenhum paciente. Não foram observados cefaléia pós-punção nem sintomas neurológicos temporários.

CONCLUSÕES: A raquianestesia com soluções hipobárica e hiperbárica proporcionou maior freqüência de unilateralidade. Após 20 minutos a solução isobárica de bupivacaína mobilizou-se no líquido cefalorraquidano (LCR), resultando em apenas $28 \%$ de raquianestesia unilateral.

Unitermos: ANESTÉSICOS: bupivacaína; CIRURGIA, Ortopédica; TÉCNICAS ANESTÉSICAS, Regional: raquianestesia unilateral.

\section{SUMMARY}

Imbelloni LE, Beato L, Gouveia MA, Cordeiro JA - Low Dose Isobaric, Hyperbaric, or Hypobaric Bupivacaine for Unilateral Spinal Anesthesia.

BACKGROUND AND OBJECTIVES: Unilateral spinal anesthesia has its advantages, especially in patients undergoing outpatient basis surgeries. Low dose, slow speed of administration, and the lateral positioning make easier the unilateral distribution in spinal anesthesia. Isobaric, hyperbaric, and hypobaric solutions of bupivacaine were compared in the unilateral spinal anesthesia in patients undergoing outpatient basis orthopedic surgeries.

METHODS: One hundred and fifty patients were randomly divided in three groups to receive $5 \mathrm{mg}$ of $0.5 \%$ isobaric bupivacaine (Iso Group), $5 \mathrm{mg}$ of $0.5 \%$ hyperbaric bupivacaine (Hyper Group), or 5 $\mathrm{mg}$ of $0.15 \%$ hypobaric bupivacaine (Hypo Group). The solutions were administered in the $L_{3}-L_{4}$ space with the patient in the lateral decubitus and remaining in this position for 20 minutes. Sensitive anesthesia was evaluated by the pin prick test. Motor blockade was determined by the modified Bromage scale. Both blockades were compared with the opposite side and among themselves.

RESULTS: There was a significant difference between the side of the surgery and the opposite side in all three groups at 20 minutes, but the frequency of unilateral spinal anesthesia was greater with the hyperbaric and hypobaric solutions. Sensitive and motor blockades were observed in 14 patients in the Iso Group, 38 patients in the Hyper Group, and 40 patients in the Hypo Group. Patients did not develop any hemodynamic changes. Postpuncture headache and transitory neurological symptoms were not observed.

CONCLUSIONS: Spinal anesthesia with hypobaric and hyperbaric solutions present a higher frequency of unilateral anesthesia. After 20 minutes, isobaric bupivacaine mobilized into cerebrospinal fluid (CSF) resulted in unilateral spinal anesthesia in only $28 \%$ of the patients.

Key Words: ANESTHETICS: bupivacaine; ANESTHETIC TECHNIQUES, Regional: unilateral spinal block; SURGERY, Orthopedics. 


\section{INTRODUÇÃO}

A diferença de densidade entre o líquido cefalorraquidiano (LCR) e as soluções de anestésicos locais é um fator que deve ser considerado para que se restrinja sua distribuição no espaço subaracnóideo. A solução de bupivacaína a 0,5\% (sem glicose) age como uma solução discretamente hipobárica quando empregada para raquianestesia ${ }^{1}$. Mantendo-se o paciente sentado por dois minutos após a injeção de anestésico isobárico, o resultado é um bloqueio mais alto que o observado em paciente colocado imediatamente em decúbito dorsal após a injeção ${ }^{2}$.

Teoricamente, o bloqueio espinal unilateral poderia ser obtido por injeção no espaço subaracnóideo de uma solução hipobárica, isobárica ou hiperbárica, injetada com o paciente em decúbito lateral, de tal forma que o anestésico formasse uma camada acima (hipobárica ou isobárica) ou abaixo (hiperbárica) da linha média. A injeção dirigida para um dos lados, assim como a distribuição da solução em função da baricidade, resultaria em algo mais, já que não há perda de anestésico por causa de turbulência, como ocorre quando de injeção rápida. A dose de 1,2 $\mathrm{mL}(6 \mathrm{mg})$ de bupivacaína a $0,5 \%$ hiperbárica tem produzido maior índice de resultado unilateral que a dose de 1,2 $\mathrm{mg}(6 \mathrm{mg})$ de solução isobárica, quando injetada lentamente através de agulha $27 \mathrm{G}$ com ponta de Whitacre e o paciente é mantido em decúbito lateral por 20 minutos ${ }^{3}$. Quando se injetaram 3,4 mL $(6,1 \mathrm{mg})$ de bupivacaína a $0,18 \%$ hipobárica nas mesmas condições, não se observou vantagem sobre o volume de 1,2 $\mathrm{mL}$ (6 mg) de bupivacaína isobárica ${ }^{4}$. Também não oferece vantagem o uso de solução de bupivacaína hiperbárica a 1\% quando se compara com a solução a $0,5 \%{ }^{5}$. Pacientes que receberam $8 \mathrm{mg}$ de bupivacaína a 0,5\% apresentaram maior incidência de bloqueio unilateral do que aqueles que receberam 8 mg da solução a $1 \%{ }^{5}$. Na opinião de vários autores, a raquianestesia unilateral é uma técnica de baixa dose e baixo fluxo, incluindo a manutenção do decúbito lateral por um período de 15 a 30 minutos, como o melhor meio de produzir o bloqueio unilateral ${ }^{6-10}$.

O objetivo deste estudo prospectivo aleatório foi avaliar a freqüência do bloqueio unilateral quando foram usadas solução isobárica, hiperbárica e hipobárica para intervenções cirúrgicas ortopédicas. Foi avaliada a efetividade do bloqueio cirúrgico, assim como sua recuperação e as variações hemodinâmicas produzidas.

\section{MÉTODO}

O protocolo foi aprovado pela Comissão de Ética da Instituição e todos os pacientes foram esclarecidos e concordaram em participar. Os critérios de exclusão foram hipovolemia, doença neurológica preexistente, distúrbios da coagulação, administração de tromboprofilaxia há menos de oito horas, infecção no local da punção, agitação e delírio, além da presença de cateter vesical de demora. Foram estudados 150 pacientes, estado físico ASA I e II, sem medicação préanestésica, com idade entre 20 e 60 anos, programados para intervenção cirúrgica ortopédica unilateral sob raquianestesia. Em todos os pacientes foi empregado garrote pneumático na coxa, inflado até a pressão máxima de 350 $\mathrm{mmHg}$. Os pacientes foram aleatoriamente alocados em três grupos de 50 pacientes. Os pacientes do Grupo Iso receberam $1 \mathrm{~mL}$ de bupivacaína a 0,5\% pura, com densidade de 0,9993 g.mL-1; os pacientes do Grupo Hiper receberam $1 \mathrm{~mL}$ bupivacaína a $0,5 \%(5 \mathrm{mg})$ com $8 \%$ de glicose com densidade de 1,021 g.mL-1; e os pacientes do Grupo Hipo receberam $3,4 \mathrm{~mL}$ de bupivacaína a $0,15 \%$ (5 mg), densidade $0,9964 \mathrm{~g} \cdot \mathrm{mL}^{-1}$ (preparada a partir de $7,5 \mathrm{mg}=1,5 \mathrm{~mL}$ de bupivacaína isobárica a $0,5 \%$ com mais $3,5 \mathrm{~mL}$ de água destilada) (Grupo Hipo). As intervenções cirúrgicas foram videoartroscopia de joelho, correção de fraturas de tíbia, tornozelo, associada ou não à lesão ligamentar e retirada de material de síntese abaixo do joelho.

Todos os pacientes tiveram um período de jejum de seis horas. Antes da anestesia foi instalada uma via venosa para hidratação com solução fisiológica a $0,9 \%$ ou Ringer com lactato e medicação durante o procedimento. Em seguida, os pacientes foram monitorizados, com ECG, freqüência cardíaca e oxímetro de pulso contínuos, e pressão arterial por método não-invasivo. Antes do bloqueio, os pacientes receberam uma dose de $1 \mu \mathrm{g} . \mathrm{kg}^{-1}$ de fentanil por via venosa. Se a pressão arterial sistólica diminuísse mais de $30 \%$ do valor inicial, seria tratada inicialmente com aumento da velocidade de hidratação. Na ausência de resposta, seria administrada uma dose de $2 \mathrm{mg}$ de etilefrina e repetida até atingir uma resposta satisfatória. A bradicardia foi definida como diminuição da freqüência cardíaca menor que 50 bpm. O tratamento seria a administração de atropina na dose de até $0,75 \mathrm{mg}$. Todos os pacientes receberam oxigênio (1 a 2 L. $\mathrm{min}^{-1}$ ) pela máscara de Hudson. $\mathrm{O}$ midazolam (1 a $2 \mathrm{mg}$ ) foi administrado após a segunda avaliação, aos 40 minutos. Analgesia venosa suplementar seria administrada se ocorresse queixa ou desconforto devido à analgesia insuficiente. A falha foi definida como ausência de analgesia na região perineal.

Usando uma lista de números aleatórios, um residente de Anestesiologia alocou os pacientes em um dos grupos de tratamento. Nos Grupos Iso e Hipo os pacientes foram colocados com o lado a ser operado voltado para cima. No Grupo Hiper eles foram colocados com o lado a ser operado voltado para baixo. Após preparo da pele com clorexedina alcoólica, foi realizada infiltração da pele com lidocaína a 1\% no ponto de punção. A punção foi realizada pela via paramediana, no espaço, $L_{3}-L_{4}$, com agulha descartável 27G Quincke (B.Braun Melsungen S.A.) sem o uso de introdutor. Uma vez obtido o fluxo de LCR, foi injetado $1 \mathrm{~mL}(5 \mathrm{mg})$ de bupivacaína isobárica ou hiperbárica a $0,5 \%$ ou $3,4 \mathrm{~mL}$ (5 mg) da solução hipobárica a $0,15 \%$ na velocidade de $1 \mathrm{~mL} . \mathrm{seg}^{-1}$. O bisel da agulha foi direcionado para o lado a ser operado em todos os grupos. Os pacientes foram man- 
tidos na mesma posição por 20 minutos antes de serem colocados em decúbito dorsal para o procedimento cirúrgico. As avaliações dos bloqueios sensitivo e motor foram realizadas por um anestesiologista que desconhecia a que grupo pertencia o paciente. O nível do bloqueio sensitivo, definido como a falta de reconhecimento de picada ou toque com o mandril da agulha usada para a sua raquianestesia, foi avaliado bilateralmente na linha médio clavicular, enquanto o bloqueio motor foi avaliado pela escala de Bromage modificada ${ }^{11}: 0=$ movimento livre dos membros inferiores; 1 = incapacidade de levantar os membros estendidos; 2 = incapacidade de flexionar os joelhos; 3 = incapacidade de movimentar os tornozelos. Os bloqueios motor e sensitivo foram avaliados em ambos os membros aos 20 , 40, 60 minutos, ao final do procedimento e comparados entre o membro a ser operado com o membro não operado. $\mathrm{A}$ duração da analgesia foi determinada pelo tempo para o retorno da sensibilidade no dermátomo correspondente ao local da punção. Os parâmetros hemodinâmicos foram avaliados com três minutos de intervalo durante os primeiros 15 minutos após a administração do anestésico. Dados relativos ao tempo de recuperação dos bloqueios sensitivo e motor, assim como tempo para a primeira micção, deambulação e condições para alta hospitalar, de acordo com critério padronizado de alta. Complicações na sala de recuperação pós-anestésica, necessidade de cateterismo vesical, dor e tratamentos administrados foram registrados por um observador. O tempo para início da deambulação foi orientado pelo cirurgião e a analgesia pós-operatória consistiu em tenoxicam $(40 \mathrm{mg})$ e dipirona $(3 \mathrm{~g})$ por via venosa. A primeira dose de analgésico foi administrada ao final do procedimento, na sala cirúrgica.

Ao sair da sala cirúrgica, os pacientes foram solicitados a registrarem sua opinião sobre a técnica. As alternativas eram "boa", "satisfatória" e "ruim". Os pacientes foram acompanhados por três dias no pós-operatório por telefone, a fim de colher informações sobre cefaléia, sintoma neurológico temporário ou dor nas costas. Cefaléia foi classificada como pós-punção da dura-máter se apresentasse característica de piora com a posição sentada, tivesse localização occipital ou frontal e aumentasse com a tosse, es- forço ou espirro. Dor nas costas foi considerada sintoma neurológico temporário (SNT) se ocorresse dor e/ou disestesia nas costas, nádegas e pernas após a recuperação, com solução dentro de 72 horas.

As médias de idade, peso, altura, IMC e duração do bloqueio foram comparadas por Análise de Variância, enquanto nível sensitivo e bloqueio motor pelo teste de Mood para medianas. $O$ vetor de probabilidades dos níveis de satisfação do paciente do Grupo Iso foi contrastado com a média dos vetores dos dois outros grupos pelo método dos quadrados mínimos ponderados, e a unilateralidade da anestesia pelo teste Qui-quadrado para amostras independentes. O nível de significância adotado foi $\alpha=0,05$.

\section{RESULTADOS}

As características dos pacientes e a duração das intervenções cirúrgicas estão apresentadas na tabela I. Não houve diferença entre os grupos com relação à idade, índice de massa corporal (IMC), peso ou duração cirúrgica (Tabela I). Os resultados da avaliação do bloqueio sensitivo estão na figura 1. Em todas as avaliações o bloqueio sensitivo do

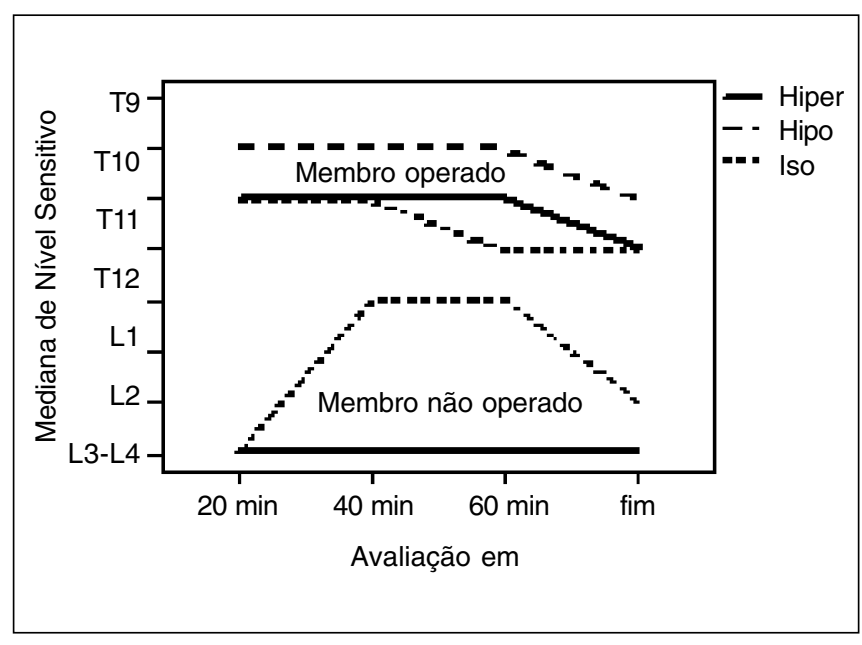

Figura 1 - Mediana do Bloqueio Sensitivo do Lado Operado e do Não Operado durante a Avaliação, de Acordo com o Grupo.

Tabela I - Características dos Pacientes, Duração Cirúrgica e dos Bloqueios (Média \pm DP)

\begin{tabular}{lcccc}
\hline Variáveis & Grupo Iso $(\mathrm{n}=50)$ & Grupo Hiper $(\mathrm{n}=50)$ & Grupo Hipo $(\mathrm{n}=50)$ & $\mathrm{p}$ \\
\hline Idade (anos) & $39,0 \pm 11,9$ & $38,7 \pm 10,7$ & $40,6 \pm 11,1$ & 0,6 \\
Peso $(\mathrm{kg})$ & $66,8 \pm 12,9$ & $69,9 \pm 11,5$ & $67,5 \pm 14,7$ & 0,47 \\
Altura (cm)* & $163,4 \pm 6,7^{\mathrm{b}}$ & $167,7 \pm 6,7^{\mathrm{a}}$ & $164,3 \pm 6,7^{\mathrm{a}, \mathrm{b}}$ & 0,008 \\
IMC & $24,9 \pm 3,9$ & $24,8 \pm 3,5$ & $24,8 \pm 3,9$ & 0,97 \\
Duração (min) & $81,00 \pm 13,62$ & $82,30 \pm 14,04$ & $84,30 \pm 14,21$ & 0,49 \\
\hline
\end{tabular}

a ba,b: a média da altura do Grupo 2 = Hiper ${ }^{(a)}$ é significativamente mais alta que a do Grupo 1 = Iso ${ }^{(b)}$, e que não há evidência de que a média do Grupo 3 = Hipo ${ }^{(a, b)}$ seja diferente da média dos dois grupos. 
membro operado foi significativamente mais alto no Grupo Hipo que nos outros dois, com $p<0,05$, assim como o bloqueio sensitivo no membro não operado foi, em todas as avaliações, exceto no final, mais alto no Grupo Iso que nos dois outros grupos, com $p<0,05$, muito mais alto no membro operado em todos os grupos em todos os momentos, a mediana das diferenças de segmentos variando de 2 a 5 $(p<0,05)$, de acordo com verificação com o estilete da aguIha. A duração média do bloqueio foi significativamente mais longa no Grupo Iso $(2,94 \pm 0,5 \mathrm{~h})$ do que no Grupo Hiper $(2,48 \pm 0,38 \mathrm{~h})$ e no Grupo Hipo $(2,3 \pm 0,36 \mathrm{~h})(\mathrm{p}<0,05)$.

Durante a manutenção da posição de decúbito lateral (20 minutos) o bloqueio sensitivo foi exclusivamente unilateral em 31 pacientes do Grupo Iso (62\%), 42 pacientes no Grupo Hiper (84\%) e 45 pacientes no Grupo Hipo (90\%). Após reposicionar o paciente em decúbito dorsal, o bloqueio sensitivo se redistribuiu para o lado não operado, e 40 minutos depois o bloqueio ainda se mantinha unilateral em 16 pacientes do Grupo Iso (32\%), 42 pacientes do Grupo Hiper $(84 \%)$ e 43 pacientes no Grupo Hipo $(86 \%)(p<0,05)$. O bloqueio sensitivo mais alto (aos 20 minutos) do Grupo Hipo foi significativamente mais alto que os outros dois grupos, que não diferiram entre si $(p<0,05)$.

A avaliação do bloqueio motor está representada na figura 2. Durante a posição lateral, o bloqueio motor foi completamente unilateral em 32 pacientes do Grupo Iso (64\%), 43 pacientes no Grupo Hiper (86\%) e 48 pacientes no Grupo Hipo (96\%). Após recolocar os pacientes em decúbito dorsal, o bloqueio motor se redistribuiu para o lado não operado, e depois de 20 minutos nessa posição ainda se manteve unilateral em 14 pacientes no Grupo Iso (28\%), 38 pacientes no Grupo Hiper (76\%) e 40 pacientes no Grupo Hipo $(80 \%)(p<0,05)$.

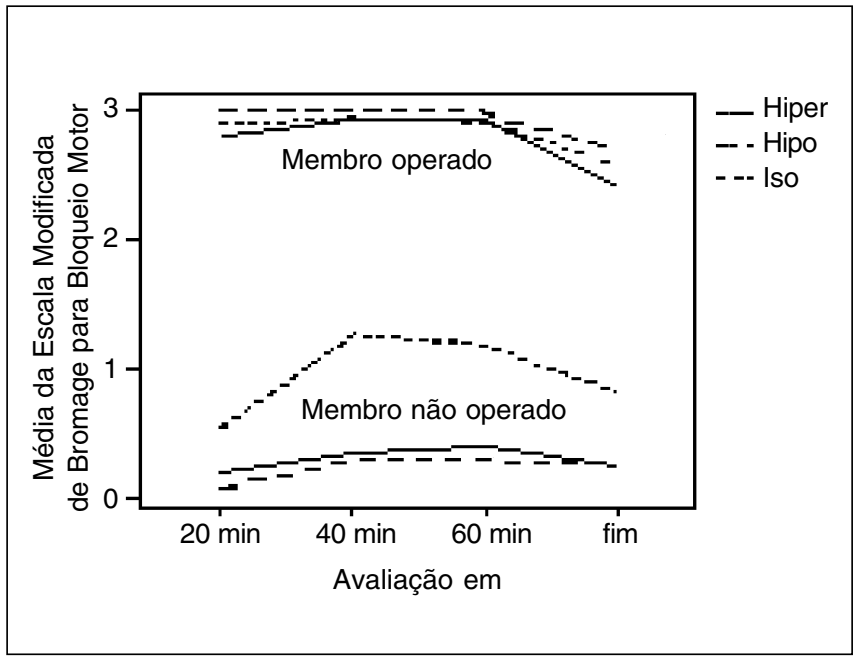

Figura 2 - Média do Bloqueio Motor no Membro Operado e no Membro Não Operado, durante as Avaliações de Acordo com o Grupo.

O bloqueio unilateral aos 20 minutos foi conseguido em $62 \%$ dos pacientes do Grupo Iso, $84 \%$ no Grupo Hiper e $86 \%$ no Grupo Hipo. Aos 40 e 60 minutos, somente $28 \%$ dos pacientes do Grupo Iso, $76 \%$ dos pacientes do Grupo Hiper e $80 \%$ dos pacientes do Grupo Hipo permaneceram com bloqueio unilateral. Nenhum paciente necessitou de complementação com anestesia geral por falha da raquianestesia. A satisfação dos pacientes com a anestesia está apresentada na tabela II. As maiores satisfações foram dos Grupos Hiper e Hipo, em comparação com o Grupo Iso $(p<0,05)$.

Nenhum dos pacientes de todos os grupos necessitou de tratamento por hipotensão arterial ou bradicardia.

Tabela II - Características dos Bloqueios Espinais

\begin{tabular}{|c|c|c|c|c|}
\hline & Grupo Iso $(n=50)$ & Grupo Hiper $(n=50)$ & Grupo Hipo $(n=50)$ & $\mathrm{p}$ \\
\hline Anestesia unilateral 20' & $31(62 \%)$ & $42(84 \%)$ & $45(90 \%)$ & 0,002 * \\
\hline Anestesia unilateral 40' & $14(28 \%)$ & $38(76 \%)$ & $40(80 \%)$ & 0,00005 * \\
\hline \multicolumn{5}{|l|}{ Aos 20' (segmentos acima do local da injeção) } \\
\hline Máximo bloqueio sensitivo & $5,0(3,0)$ ** & $5,0(2,0) * *$ & $6,0(2,0)$ ** & $0,002 * * *$ \\
\hline Duração do bloqueio $(\mathrm{h})$ & $2,94 \pm 0,50$ & $2,31 \pm 0,36$ & $2,48 \pm 0,38$ & 0,0005 \\
\hline Satisfação dos Pacientes & & & & 0,00005 * \\
\hline Anestesia ruim & 16 & 03 & 03 & \\
\hline Anestesia boa & 8 & 06 & 06 & \\
\hline
\end{tabular}

* Método dos quadrados mínimos ponderados para testar o contraste "Iso-(Hiper e Hipo) / 2" em todas as respostas, com três amostras aleatórias independentes.

** Mediana (amplitude interquartílica).

${ }^{* * *}$ Teste de Mood para medianas. 
Nenhum paciente apresentou cefaléia pós-punção da dura-máter ou retenção urinária. Não houve queixa de dor nas costas, nas nádegas ou nas pernas nos três dias subseqüentes.

\section{DISCUSSÃO}

Neste estudo, $5 \mathrm{mg}$ de bupivacaína hiperbárica e hipobárica proporcionaram adequada raquianestesia unilateral para intervenção cirúrgica de membro inferior. Maior número de pacientes teve bloqueio unilateral com baixa dose de bupivacaína hiperbárica $(76 \%)$ e hipobárica $(80 \%)$ do que com a bupivacaína isobárica (28\%) durante o decúbito dorsal. Os pacientes ficaram muito satisfeitos com a técnica e não houve alterações hemodinâmicas.

$\mathrm{O}$ objetivo da raquianestesia unilateral foi induzir um bloqueio motor unilateral, melhorando o conforto do paciente, já que o bloqueio motor bilateral prolongado pode ser inconveniente, além de prevenir alterações hemodinâmicas. Vários fatores têm sido relacionados com resultados insatisfatórios da raquianestesia unilateral, incluindo a posição do paciente, o desenho da agulha, a velocidade da injeção, a quantidade de anestésico local injetada e a densidade da solução empregada em relação à do LCR ${ }^{12}$. Embora se mantenha o paciente na posição lateral após a injeção, as soluções isobárica, hiperbárica e hipobárica podem influenciar a distribuição do anestésico no LCR, e o tempo de permanência na posição lateral é difícil de ser definido e é influenciado pela dose do anestésico local. Grandes doses (12 a 20 mg de bupivacaína) mostraram relevante migração do bloqueio, mesmo após uma hora em decúbito lateral ${ }^{13}$, enquanto pequenas doses (5 a $8 \mathrm{mg}$ bupivacaína) resultaram em bloqueio restrito somente após 10 a 15 minutos na posição lateral ${ }^{14}$. O melhor resultado unilateral ocorreu quando se utilizou baixa dose de bupivacaína hiperbárica ou hipobárica e o paciente foi mantido em decúbito lateral por 15 a 20 minutos ${ }^{15}$. Previamente usou-se baixa dose de bupivacaína hipobárica a $0,15 \%$ (5 mg) para intervenção cirúrgica ortopédica e os resultados indicaram que o melhor bloqueio unilateral se obtém quando o paciente se mantém na posição lateral por pelo menos 20 minutos ${ }^{10}$. Neste estudo, o local de punção e a dose foram constantes e todos os pacientes permaneceram na posição lateral por 20 minutos. A bupivacaína tem longa duração de ação. A densidade da bupivacaína isobárica $0,5 \%$ a $37^{\circ} \mathrm{C}$ é $0,9993 \mathrm{~g} \cdot \mathrm{mL}^{-1}{ }^{16}$. Quando se optar por solução hiperbárica, a bupivacaína a $0,5 \%$ com glicose a $8 \%$ pode ser usada, e sua densidade em temperatura ambiente é de $1,0247{ }^{16}$. Quando a opção for por solução hipobárica, a bupivacaína a $0,15 \%$ é sugerida ${ }^{10}$ (1,5 $\mathrm{mL}$ de bupivacaína isobárica a $0,5 \%$ e $3,5 \mathrm{~mL}$ de água destilada estéril para preparar $5 \mathrm{~mL}$ de bupivacaína a $0,15 \%$ ) com densidade de $0,996 \mathrm{~g} \cdot \mathrm{mL}^{-1}$. A posição do paciente durante e imediatamente após a injeção do anestésico local influencia a distribuição do fármaco no espaço leptomeníngeo. Se for empregada uma solução mais den- sa ou menos densa que o LCR é, pelo menos teoricamente, possível controlar a distribuição do bloqueio espinal, porque a diferença do peso específico entre a solução hiperbárica e o LCR é maior que as diferenças entre as soluções hiperbárica e isobárica, e o uso da solução hiperbárica oferece um resultado mais previsível ${ }^{15}$. Este estudo mostrou que os Grupos Hiper (76\%) e Hipo (80\%) tiveram resultado de bloqueios sensitivo e motor similar em todas as avaliações, enquanto o grupo isobárico teve apenas $62 \%$ de unilateralidade aos 20 minutos que diminuiu para $28 \%$ ao ser reposicionado em decúbito dorsal, o que está de acordo com trabalhos anteriores ${ }^{15,17}$.

O fator mais importante que influenciou a distribuição unilateral da anestesia raquidiana parece ser a dose do anestésico local empregada. A redução da dose é crucial para restringir o bloqueio a um dos lados. Contudo, uma redução extrema pode aumentar o índice de falha da raquianestesia ${ }^{18}$. Neste estudo foram usados $5 \mathrm{mg}$ de bupivacaína em todos os grupos e foi obtido resultado satisfatório com as três soluções para bloqueio unilateral, sem uma falha sequer. $O$ aumento da dose aumenta o tempo para recuperação do bloqueio ${ }^{19}$.

Grandes variações em volume e concentração de anestésico local têm pequeno papel em sua dispersão no canal leptomeníngeo ${ }^{20}$, enquanto a quantidade total de moléculas injetadas no canal espinal tem um papel mais importante. Neste estudo, com uma dose fixa de $5 \mathrm{mg}$ em todos os grupos, o volume de bupivacaína isobárica e hiperbárica foi de $1 \mathrm{~mL}$, enquanto o volume de bupivacaína hipobárica foi de $3,4 \mathrm{~mL}$. O maior volume resultou em uma dispersão maior da anestesia.

Três tipos de agulha estão atualmente em uso: o modelo ponta de lápis (Whitacre e Sprotte), a ponta de lápis atraumática modificada $\left(\right.$ Atraucan $^{\circledR}$ ) e a agulha de ponta cortante (tipo Quincke). Alto nível de sucesso em raquianestesia unilateral tem sido relatado com agulhas 25G Whitacre ${ }^{20}$ e 29G Quincke ${ }^{21}$. O calibre da agulha parece estar mais especificamente relacionado com a incidência de efeitos colaterais, como cefaléia e dor lombar. $O$ índice de sucesso em $61 \%$ (92/150 pacientes) submetidos a raquianestesia unilateral com agulha 27G Quincke demonstrou que pode ser boa indicação para esse tipo de bloqueio espinal, além de ter menor preço que as demais.

O bloqueio unilateral já foi relatado com baixa dose de bupivacaína hiperbárica ou hipobárica quando os pacientes foram mantidos 15 a 30 minutos na posição lateral. O nível do bloqueio com essas pequenas doses não parece se modificar após mudanças de decúbito mais tardias. Esse fato foi confirmado nessa observação com pequena dose de solução hiperbárica e hipobárica de bupivacaína mantendose o paciente na posição lateral por 20 minutos. Quando é empregada baixa dose de bupivacaína isobárica, o nível da unilateralidade se modifica pela movimentação, mesmo após 40 minutos da injeção. 0 mesmo ocorre quando doses maiores de bupivacaína hiperbárica ou isobárica são usa- 
das ${ }^{22,23}$. O resultado de $76 \%$ de raquianestesia unilateral com solução hiperbárica de bupivacaína é similar aos $68 \%{ }^{24}$ e $83 \%{ }^{15}$ já relatados; o resultado de $80 \%$ de raquianestesia unilateral com solução hipobárica de bupivacaína a 0,15\% é maior que os $52 \%{ }^{4}$ e $24 \%{ }^{24}$ já mencionados, e o resultado de $28 \%$ de unilateralidade com a solução isobárica é menor que os $37 \%{ }^{15}$ já publicados.

Pequena dose de anestésico local injetada no espaço subaracnóideo provoca mínimas alterações hemodinâmicas, com grande estabilidade cardiovascular. A hipotensão arterial é uma complicação da raquianestesia, que ocorre em até $33 \%$ dos pacientes quando doses maiores são empregadas ${ }^{25}$. O bloqueio unilateral, com qualquer das soluções, causa menos hipotensão arterial. Quando se comparam as alterações hemodinâmicas que ocorrem com os bloqueio unilateral e bilateral com a mesma dose de bupivacaína hiperbárica (8 $\mathrm{mg})$, as freqüências de hipotensão arterial foram $22,4 \%$ e $5 \%$, respectivamente ${ }^{26}$. Com $7,5 \mathrm{mg}$ de bupivacaína, hipotensão arterial foi observada em 1/25 pacientes no Grupo Hiper e 1/25 pacientes no Grupo Hipo, embora nenhum dos pacientes recebesse vasopressor profilático ou infusão venosa ${ }^{24}$. Neste estudo com $5 \mathrm{mg}$ de bupivacaína, nenhum dos pacientes desenvolveu hipotensão arterial.

Concluindo, $5 \mathrm{mg}$ de bupivacaína isobárica, hiperbárica e hipobárica promoveram adequada anestesia para intervenção cirúrgica ortopédica com alta satisfação dos pacientes deste estudo. Embora o bloqueio unilateral tenha sido mais freqüente nos grupos que receberam solução de bupivacaína hiperbárica (76\%) e hipobárica (80\%), não houve diferença entre eles. Diferente das soluções hiperbárica e hipobárica, a solução isobárica de bupivacaína se move no LCR mesmo após 20 minutos, mantendo-se o bloqueio unilateral em apenas $28 \%$ dos pacientes.

\section{Low Dose Isobaric, Hyperbaric, or Hypobaric Bupivacaine for Unilateral Spinal Anesthesia}

Luiz Eduardo Imbelloni, TSA, M.D.; Lúcia Beato, TSA, M.D.; Marildo A Gouveia, TSA, M.D.; José Antônio Cordeiro, M.D.

\section{INTRODUCTION}

The difference in density between the cerebrospinal fluid (CSF) and local anesthetics solutions should be considered to restrict their distribution to the subarachnoid space. The solution of $0.5 \%$ bupivacaine (without glucose) behaves as a discretely hypobaric solution when used in spinal anesthesia ${ }^{1}$. Maintaining the patient in the sitting position for two minutes after the administration of the isobaric anesthetic results in a higher blockade than if the patient were placed in the dorsal decubitus immediately after the injection ${ }^{2}$.

In theory, unilateral spinal block can be achieved adminis- tering a hypobaric, isobaric, or hyperbaric solution in the subarachnoid space with the patient in the lateral decubitus, so the anesthetic forms a layer above (hypobaric or isobaric) or below (hyperbaric) de midline. Thus, the injection directed to one of the sides, with the distribution of the solution depending on its specific gravity, would result in something else, since there is no loss of anesthetic solution secondary to turbulence, which happens with fast administration. The dose of $1.2 \mathrm{~mL}(6 \mathrm{mg})$ of $0.5 \%$ hyperbaric bupivacaine has had a greater incidence of unilateral anesthesia than $1.2 \mathrm{~mL}$ (6 $\mathrm{mg}$ ) of the isobaric solution, when it is injected slowly through a $27 \mathrm{G}$ Whitacre point needle and the patient remains in the lateral decubitus for 20 minutes ${ }^{3}$. When $3.4 \mathrm{~mL}$ $(6.1 \mathrm{mg}) \quad 0.18 \%$ hypobaric bupivacaine were injected under the same conditions, it showed no advantage over the 1.2 $\mathrm{mL}(6 \mathrm{mg})$ of isobaric bupivacaine ${ }^{4}$. The use of $1 \%$ hyperbaric bupivacaine has no advantage over the $0.5 \%$ solution ${ }^{5}$. Patients who received $8 \mathrm{mg}$ of $0.5 \%$ bupivacaine presented a greater incidence of unilateral blockade than those who received $8 \mathrm{mg}$ of the $1 \%$ solution ${ }^{5}$. According to several authors, unilateral spinal anesthesia is a low dose and low flow technique, including the maintenance of the lateral decubitus for 15 to 30 minutes ${ }^{6-10}$ as the best way of producing unilateral blockade.

The objective of this random, prospective study was to evaluate the frequency of the unilateral blockade when isobaric, hyperbaric, and hypobaric solutions were used for orthopedic surgeries. The efficacy of the blockade, as well as its recovery and hemodynamic changes, were evaluated.

\section{METHODS}

The study protocol was approved by the Ethics Committee of the institution and the procedures were explained to the patients who agreed to participate. Exclusion criteria included hypovolemia, preexisting neurological disease, coagulation disorders, administration of thrombosis prophylaxis less than eight hours prior to the procedure, infection at the puncture site, agitation and delirium, and the presence of vesical catheter. One hundred and fifty patients, ages between 20 and 60 years, ASA physical state I and II, who did not receive premedication, scheduled for unilateral orthopedic surgery under spinal anesthesia participated in the study. A sphygmomanometer was placed on the thigh of every patient and inflated to $350 \mathrm{mmHg}$. Patients were randomly divided in three groups of 50 patients. Patients in the Iso Group received $1 \mathrm{~mL} 0.5 \%$ pure bupivacaine with a density of $0.9993 \mathrm{~g} \cdot \mathrm{mL}^{-1}$; patients in the Hyper Group received $1 \mathrm{~mL}$ of $0.5 \%$ bupivacaine $\left(5 \mathrm{mg}\right.$ ) with $8 \%$ glucose, with a density of $1.021 \mathrm{~g} \cdot \mathrm{mL}^{-1}$; and patients in the Hypo Group received $3.4 \mathrm{~mL}$ of $0.15 \%$ bupivacaine $\left(5 \mathrm{mg}\right.$ ), with a density of $0.9964 \mathrm{~g} \cdot \mathrm{mL}^{-1}$ (prepared from $7.5 \mathrm{mg}=1.5 \mathrm{~mL} 0.5 \%$ isobaric bupivacaine with $3.5 \mathrm{~mL}$ of distilled water). The surgeries included knee videoarthroscopy, correction of tibial and ankle fractures, associated or not with ligament lesion, and removal of 
synthesis material below the knee. Every patient was submitted to a 6-hour fasting period prior to the procedure. Before the anesthesia, a peripheral venous access was placed for hydration with $0.9 \%$ normal saline or Ringer's lactate and administration of medications during the procedure. Patients were monitored with continuous ECG, heart rate, and pulse oxymeter, and non-invasive blood pressure. Before the blockade, patients received a dose of $1 \mu \mathrm{gg} \cdot \mathrm{kg}^{-1}$ of intravenous fentanyl. If systolic blood pressure showed a reduction greater than $30 \%$ of baseline values, it would initially be treated increasing the speed of the hydration. If the patient did not respond, $2 \mathrm{mg}$ of etilephrine would be administered and the dose repeated until a satisfactory response was achieved. Bradycardia was defined as a reduction of heart rate below $50 \mathrm{bpm}$. It would be treated with the administration of atropine up to a dose of $0.75 \mathrm{mg}$. Every patient received oxygen ( 1 to 2 L. $\mathrm{min}^{-1}$ ) by Hudson mask. Midazolam (1 to $2 \mathrm{mg}$ ) was administered after the second evaluation, at the 40-minute mark. Supplemental intravenous analgesia would be administered if the patient complained of pain or discomfort. Failure of analgesia was defined as absence of analgesia in the perineal region.

Using a list of random numbers, an Anesthesiology resident assigned the patients in one of the treatment groups. The patients in the Iso and Hypo Groups were placed on the side that was not to be operated. The patients in the Hyper Group were placed on the side to be operated on. After cleansing the skin with alcoholic chlorhexidine, the skin of the puncture site was infiltrated with $1 \%$ lidocaine. The puncture was paramedian, at the $L_{3}-L_{4}$ space, with a discardable $27 \mathrm{G}$ Quincke needle (B. Braun Melsungen S.A.) without the introducer. Once there was flow of CSF, $1 \mathrm{~mL}(5 \mathrm{mg})$ of isobaric, $0.5 \%$ hyperbaric, or $3.4 \mathrm{~mL}(5 \mathrm{mg}), 0.15 \%$ hypobaric bupivacaine was injected at $1 \mathrm{~mL} . \mathrm{seg}^{-1}$. The bevel of the needle was directed to the side to be operated in every patient. Patients remained in the same position for 20 minutes before being placed in the dorsal decubitus for the surgery. Assessment of sensitive and motor blockades was done by an anesthesiologist who was not aware to which group the patient belonged. The level of the sensitive blockade, defined as a lack of pinprick recognition or the touch with the mandril of the puncture needle, was determined bilaterally at the midclavicular line, while the motor blockade was determined by the modified Bromage scale ${ }^{11}: 0=$ moving the inferior limbs freely; $1=$ unable to raise extended limbs; $2=$ unable to bend the knees; and $3=$ unable to move the ankles. Motor and sensitive blockades were evaluated on both lower limbs 20,40, and 60 minutes after the anesthesia and at the end of the procedure, and the limb to be operated on was compared to the opposite limb. The length of analgesia was determined by the time to regain sensitivity in the dermatome corresponding to the puncture site. Hemodynamic parameters were evaluated at three-minute intervals for the first 15 minutes after the administration of the anesthetic. Data regarding the time of recovery of sensitive and motor blockades, as well the first urine voided, ambulation, and conditions for hospital discharge were recorded. Complications in the recovery room, the need for a vesical catheter, pain, and treatment administered were recorded by an observer. The time to begin ambulation was oriented by the surgeon and the postoperative analgesia consisted of intravenous tenoxicam $(40 \mathrm{mg})$ and dypirone $(3 \mathrm{~g})$. The first dose of analgesic was administered at the end of the surgical procedure, in the surgical room.

Upon leaving the surgical room, patients were requested to record their opinion about the technique employed. The choices included good, satisfactory, and bad. Patients were followed-up for three days postoperatively over the phone to gather information regarding the presence of headache, temporary neurological symptoms, or back pain. Headache was classified as dura mater postpuncture if it worsened when the patient was in the sitting position, were located in the occipital or frontal region, and increased with coughing, strain, or sneezing. Back pain was considered a transitory neurological symptom (TNS) if the patient experienced pain and/or decreased sensitivity in the back, buttocks, and legs after recovery, which resolved within 72 hours.

Mean age, weight, height, BMl, and duration of the blockade were compared by Analysis of Variance, while the level of the sensitive and motor blockades was analyzed by the Mood test for medians. The probability vectors of the satisfaction levels of the patients in the Iso Group was compared with the mean of the vectors of the other two groups by the pondered minimal quadrature method, and the unilaterality of the anesthesia was analyzed by the Chi-square test for independent samples. A level of significance of $\alpha=0.05$ was adopted.

\section{RESULTS}

Table I shows the characteristics of the patients and the duration of the surgeries. There were no differences among the groups regarding age, body mass index (BMI), weight, or duration of the surgical procedure (Table I).

Figure 1 shows the evaluation of the sensitive blockade. The level of the sensitive blockade on the limb operated on was higher in the Hypo Group than in the other two, which was statistically significant $(p<0.05)$; the level of the sensitive blockade in the opposite limb was higher in the Iso Group, except at the end of the procedure, with a $p<0.05$; the level of the sensitive blockade was much higher in the operated limb in all three groups at every moment; and the median of the differences of the segments varied from 2 to $5(p<0.05)$, according to the pinprick evaluation. The mean duration of the blockade was longer in the Iso Group $(2.94 \pm 0.5 \mathrm{~h})$ than in the Hyper Group (2.48 $\pm 0.38 \mathrm{~h})$ and Hypo Group $(2.3 \pm 0.36 \mathrm{~h})$ $(p<0.05)$.

During the time the patient was kept in the lateral decubitus (20 minutes), the sensitive blockade was exclusively unilateral in 31 patients in the Iso Group (62\%), 42 patients in the Hyper Group (84\%), and 45 patients in the Hypo Group (90\%). 
Table I - Characteristics of the Patients, Duration of the Surgery and of the Blockades (Mean \pm SD)

\begin{tabular}{lcccc}
\hline Variables & Iso Group $(\mathrm{n}=50)$ & Hyper Group $(\mathrm{n}=50)$ & Hypo Group $(\mathrm{n}=50)$ & $\mathrm{p}$ \\
\hline Age (years) & $39.0 \pm 11.9$ & $38.7 \pm 10.7$ & $40.6 \pm 11.1$ & 0.6 \\
Weight $(\mathrm{kg})$ & $66.8 \pm 12.9$ & $69.9 \pm 11.5$ & $67.5 \pm 14.7$ & 0.47 \\
Height $(\mathrm{cm})^{\star}$ & $163.4 \pm 6.7^{\mathrm{b}}$ & $167.7 \pm 6.7^{\mathrm{a}}$ & $164.3 \pm 6.7^{\mathrm{a}, \mathrm{b}}$ & 0.008 \\
BMl & $24.9 \pm 3.9$ & $24.8 \pm 3.5$ & $24.8 \pm 3.9$ & 0.97 \\
Duration $(\mathrm{min})$ & $81.00 \pm 13.62$ & $82.30 \pm 14.04$ & $84.30 \pm 14.21$ & 0.49 \\
\hline
\end{tabular}

a ba,b: the mean height in Group $2=$ Hyper $^{(a)}$ is significantly higher than Group $1=$ Iso(b), and there are no evidences that the mean in Group $3=$ $\mathrm{Hypo}^{(\mathrm{a}, \mathrm{b})}$ is different from the other two groups.

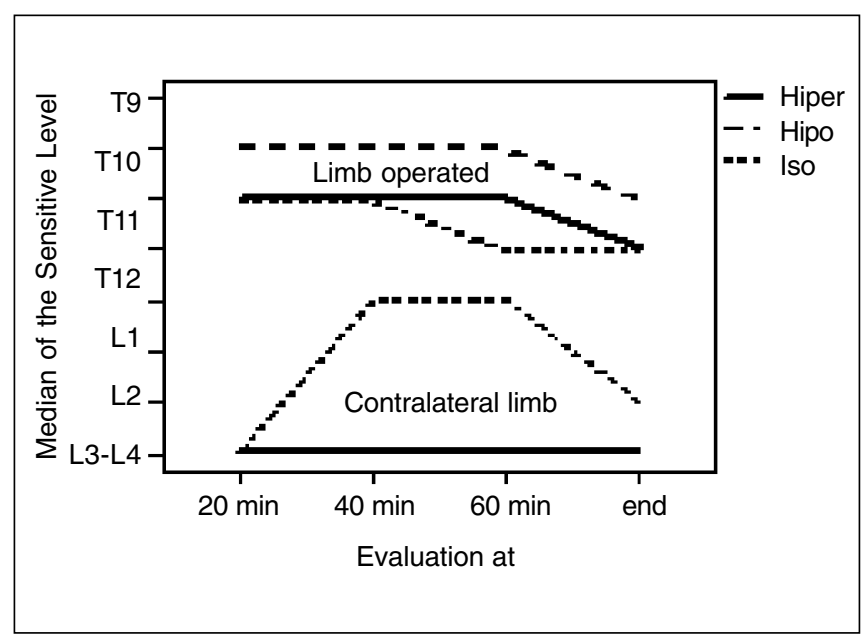

Figure 1 - Median of the Sensitive Blockade on the Side Operated on and the Contralateral Side During the Evaluation, According to the Group.

After placing the patient in the dorsal decubitus, the sensitive blockade spread to the other side, and after 40 minutes the blockade remained unilateral in 16 patients in the Iso Group (32\%), 42 patients in the Hyper Group (84\%), and 43 patients in the Hypo Group (86\%) ( $p<0.05)$. The highest sensitive blockade (at 20 minutes) in the Hypo Group was significantly higher than in the other two groups, which did not show any differences among them ( $p<0.05)$.

Figure 2 shows the evaluation of the motor blockade. While the patients were in the lateral decubitus, the motor blockade was completely unilateral in 32 patients in the Iso Group (64\%), 43 patients in the Hyper Group (86\%), and 48 patients in the Hypo Group (96\%). After replacing the patients in the dorsal decubitus, the motor blockade spread to the other side, and after 20 minutes in this position, it remained unilateral in 14 patients in the Iso Group (28\%), 38 patients in

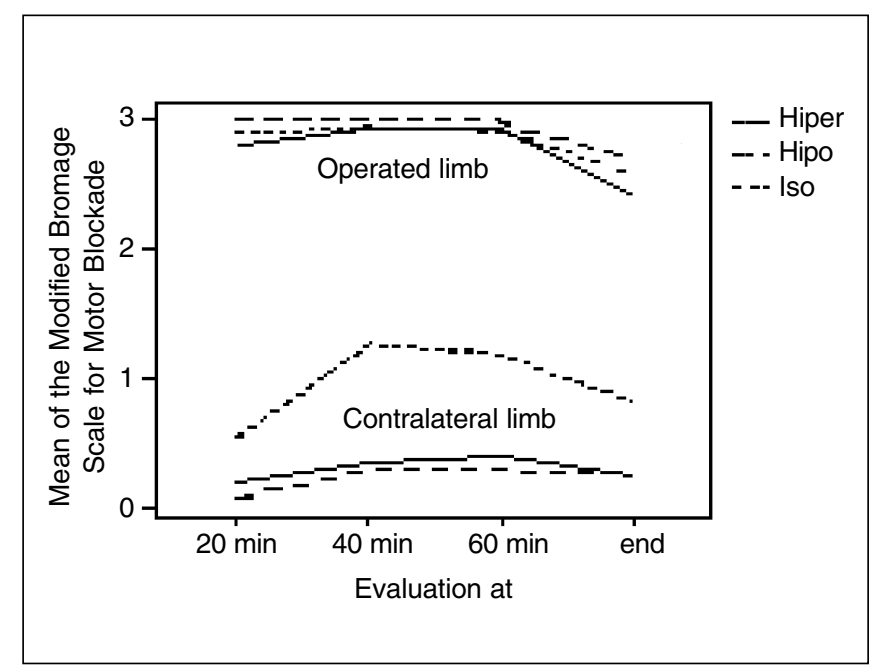

Figure 2 - Mean of the Motor Blockade in the Operated Limb and in the Contralateral Limb During the Evaluations According to the Group.

the Hyper Group (76\%), and 40 patients in the Hypo Group $(80 \%)(p<0.05)$.

At 20 minutes, $62 \%$ of the patients in the Iso Group, $84 \%$ in the Hyper Group, and $86 \%$ in the Hypo Group presented unilateral blockade. At 40 and 60 minutes, $28 \%$ of the patients in the Iso Group, $76 \%$ in the Hyper Group, and $80 \%$ in the Hypo Group remained with unilateral blockade. Patients did not need complementary general anesthesia secondary to failure of the spinal anesthesia. Table II shows patient satisfaction with the techniques. Patients in the Hyper and Hypo Groups presented the highest incidence of satisfaction $(p<0.05)$.

There were no cases of hypotension or bradycardia. Also, no cases of postpuncture headache or urinary retention were recorded. There were no complaints of back pain, pain in the buttocks or in the legs in the next three days. 
Table II - Characteristic of the Spinal Blocks

\begin{tabular}{|c|c|c|c|c|}
\hline & Iso Group $(n=50)$ & Hyper Group $(n=50)$ & Hypo Group $(n=50)$ & $\mathrm{p}$ \\
\hline Unilateral anesthesia 40' & $14(28 \%)$ & $38(76 \%)$ & $40(80 \%)$ & $0.00005^{\star}$ \\
\hline \multicolumn{5}{|l|}{ At 20' (segments above injection site) } \\
\hline Maximal sensitive blockade & $5.0(3.0)^{\star *}$ & $5.0(2.0)^{\star *}$ & $6.0(2.0)^{\star *}$ & $0.002^{\star * *}$ \\
\hline Patient Satisfaction & & & & $0.00005^{\star}$ \\
\hline Bad anesthesia & 16 & 03 & 03 & \\
\hline Good anesthesia & 8 & 06 & 06 & \\
\hline
\end{tabular}

* Pondered minimal quadrature method to test the contrast "Iso-(Hyper and Hypo) / 2" in all responses with three random independent samples.

${ }^{* *}$ Median (amplitude between percentiles).

${ }^{\star * *}$ Mood test for medians.

\section{DISCUSSION}

In this study, $5 \mathrm{mg}$ of hyperbaric and hypobaric bupivacaine provided adequate unilateral spinal anesthesia for surgeries of the lower limbs. More patients obtained unilateral blockade with low dose of hyperbaric (76\%) and hypobaric (80\%) bupivacaine than with the isobaric (28\%) bupivacaine during the dorsal decubitus. Patients were very satisfied with the technique and there were no hemodynamic changes.

The objective of the unilateral spinal anesthesia was to induce unilateral motor blockade, improving patient comfort, since prolonged bilateral motor blockade can be inconvenient, and to prevent hemodynamic changes. Several factors have been related with poor results of unilateral spinal anesthesia, including the position of the patient, the design of the needle, the speed of administration, the amount of local anesthetic injected, and the density of the anesthetic solution compared to the CSF ${ }^{12}$. Although the patient is kept in the lateral decubitus after the injection, the isobaric, hyperbaric, and hypobaric solutions can influence the distribution of the anesthetic in the CSF, and the length of time in the lateral position is difficult to be defined, being influenced by the dose of the local anesthetic. Large doses (12 to $20 \mathrm{mg}$ of bupivacaine) are related with important migration of the blockade, even after one hour in the lateral decubitus ${ }^{13}$, while small doses (5 to $8 \mathrm{mg}$ of bupivacaine) resulted in a restricted blockade only after 10 to 15 minutes in the lateral decubitus ${ }^{14}$. The best unilateral result was obtained with low dose hyperbaric or hypobaric bupivacaine, keeping the patient in the lateral decubitus for 15 to 20 minutes ${ }^{15}$. Low dose of $0.15 \%$ hypobaric bupivacaine $(5 \mathrm{mg}$ ) was used previously in orthopedic surgeries and the results indicated that the best unilateral blockade was obtained when the patient remained in the lateral decubitus for at least 20 minutes ${ }^{10}$. In this study, the puncture site and the dose were constant, and every patient remained in the lateral decubitus for 20 minutes.

Bupivacaine is a long-acting drug. The density of the $0.5 \%$ isobaric bupivacaine at $37^{\circ} \mathrm{C}$ is $0.9993 \mathrm{~g} \cdot \mathrm{mL}^{-1}{ }^{16}$. When using the hyperbaric solution, $0.5 \%$ bupivacaine with $8 \%$ glucose can be used, and its density at room temperature is $1.0247{ }^{16}$. When using the hypobaric solution, $0.15 \%$ bupivacaine, with a density of 0.996 g. mL ${ }^{-1}$, is suggested ${ }^{10}(1.5 \mathrm{~mL} 0.5 \%$ isobaric bupivacaine and $3.5 \mathrm{~mL}$ sterile distilled water to prepare $5 \mathrm{~mL}$ of $0.15 \%$ bupivacaine). The position of the patient during and immediately after the injection of the local anesthetic influences the distribution of the drug in the leptomeningeal space. If a solution that is more or less dense than the CSF is used, it is possible, at least in theory, to control the distribution of the spinal blockade, because the difference of the specific gravity between the hyperbaric solution and the CSF is greater than the differences between the hyperbaric and isobaric solutions. Therefore, the use of the hyperbaric solution has a more predictable result ${ }^{15}$. This study showed that the Hyper $(76 \%)$ and Hypo $(80 \%)$ Groups had similar sensitive and motor blockades in every evaluation, while the isobaric group presented only $62 \%$ of patients with unilateral blockade at 20 minutes, which decreased to $28 \%$ when they were placed in the dorsal decubitus, which agrees with other studies in the literature ${ }^{15,17}$.

The dose of the local anesthetic seems to be the most important factor influencing the unilateral distribution of spinal anesthesia. A reduction of the dose is critical to restrict the blockade to one side. However, an excessive reduction could increase the failure index of the spinal anesthesia ${ }^{18}$. In this study, we used $5 \mathrm{mg}$ of bupivacaine in all three groups, 
obtaining satisfactory results with the three solutions for the unilateral blockade, without any failures. Increasing the dose increases the recovery time of the blockade ${ }^{19}$.

Large variations in volume and concentration of the local anesthetic have little influence in its dispersion in the leptomeningeal channel ${ }^{20}$, while the total amount of molecules injected in the spinal canal is more important. In this study, using a fixed dose of $5 \mathrm{mg}$ in all three groups, the volume of isobaric and hyperbaric bupivacaine corresponded to $1 \mathrm{~mL}$, and the volume of hypobaric bupivacaine corresponded to $3.4 \mathrm{~mL}$. The greater volume resulted in greater dispersion of the anesthesia.

Three types of needles are currently being used: the pencil tip model (Whitacre and Sprotte), the modified non-traumatic pencil tip (Atraucan ${ }^{\circledR}$ ), and the cutting tip (Quincke type). A high success rate of unilateral spinal anesthesia has been reported using 25G Whitacre ${ }^{20}$ and 29G Quincke ${ }^{21}$ needles. The caliber of the needle seems to be more specifically related with the incidence of side effects, such as headache and lumbar pain. The success rate in $61 \%$ (92/150 patients) of the patients submitted to unilateral spinal anesthesia with a 27G Quincke needle demonstrated that it can be a good indication for this type of spinal block, besides being less expensive than the others. Unilateral block has been reported with low doses of hyperbaric or hypobaric bupivacaine when patients remained in the lateral decubitus for 15 to 30 minutes. The level of the blockade with these low doses does not seem to change after the change in decubitus. This was confirmed in this study using small doses of hyperbaric and hypobaric bupivacaine and keeping the patients in the lateral decubitus for 20 minutes. When low dose of isobaric bupivacaine is used, the level of the unilaterality changes when the decubitus changes, even 40 minutes after the injection. The same happens when higher doses of hyperbaric or isobaric bupivacaine are used ${ }^{22,23}$. The result of $76 \%$ of unilateral spinal anesthesia with the hyperbaric solution of bupivacaine is similar to the $68 \%$ and $83 \%{ }^{15}$ reported in the literature; the $80 \%$ incidence of unilateral spinal anesthesia using $0.15 \%$ hypobaric bupivaciane is greater than the $52 \%{ }^{4}$ and $24 \%{ }^{24}$ reported in the literature, while the $28 \%$ incidence of unilateral spinal anesthesia using the isobaric solution is smaller than the $37 \%{ }^{15}$ reported in the literature. Small amounts of local anesthetic injected in the subarachnoid space causes minimal hemodynamic changes, maintaining great cardiovascular stability. Hypotension is a complication of spinal anesthesia, which affects $33 \%$ of the patients when higher doses are used ${ }^{25}$. The unilateral blockade with either one of the three solutions causes less hypotension. When hemodynamic changes secondary to unilateral and bilateral blockade with the same dose of hyperbaric bupivacaine ( $8 \mathrm{mg}$ ) are compared, the frequency of hypotension are $22.4 \%$ and $5 \%$, respectively ${ }^{26}$. With $7.5 \mathrm{mg}$ of bupivaciane, hypotension was observed in 1/25 patients in the Hyper Group, and 1/25 patients in the Hypo Group, although prophylactic vasopressor or intravenous infusion were not administered ${ }^{24}$. In this study, there were no cases of hypotension using $5 \mathrm{mg}$ of bupivacaine.

To conclude, in this study, $5 \mathrm{mg}$ of isobaric, hyperbaric, and hypobaric bupivacaine promoted adequate anesthesia for orthopedic surgeries and high patient satisfaction. Although the unilateral blockade was more frequent in the groups who received hyperbaric (76\%) and hypobaric (80\%) bupivacaine, there were no differences between them. Contrary to the hyperbaric and hypobaric solutions, isobaric bupivacaine moves in the CSF even after 20 minutes, maintaining the unilateral blockade in only $28 \%$ of the patients.

\section{REFERÊNCIAS - REFERENCES}

01. Logan MR, McLure JH, Wildsmith JAW - Plain bupivacaine with epinephrine. Anesthesia and analgesia. Br J Anaesth, 1986; 58:292-296.

02. Tuominen M, Kalso E, Rosenberg PH - Effects of posture on the spread of spinal anaesthesia with isobaric $0.75 \%$ or $0.5 \%$ bupivacaine. Br J Anaesth, 1982;54:313-318.

03. Casati A, Fanelli G, Cappelleri G et al. - Does speed of intrathecal injection affect the distribution of $0.5 \%$ hyperbaric bupivacaine? Br J Anaesth, 1998;81:355-357.

04. Kuusniemi KS, Pihlajamaki KK, Pitkanen MT et al. - A low-dose hypobaric bupivacaine spinal anesthesia for knee arthroscopies. Reg Anesth, 1997;22:534-538.

05. Hartmann $\mathrm{B}$, Junger $\mathrm{A}$, Klasen $\mathrm{J}$ et al. - The incidence and risk factors for hypotension after spinal anesthesia induction: an analysis of automated data collection. Anesth Analg, 2002; 94:1521-1529.

06. Pittoni G, Toffoletto F, Calcarella G et al. - Spinal anesthesia in outpatient knee surgery: 22-gauge versus 25-gauge Sprotte needle. Anesth Analg, 1995;81:73-79.

07. Casati A, Fanelli G, Berti M et al. - Cardiac performance during unilateral lumbar spinal block after crystalloid preaload. Can J Anaesth, 1997;44:623-628.

08. Vaghadia $\mathrm{H}-$ Spinal anaesthesia for outpatients: controversies and new techniques. Can J Anaesth, 1998;45:R64-R75.

09. Enk D - Unilateral spinal anaesthesia: gadget or tool? Curr Opin Anaesthesiol, 1998;11:511-515.

10. Imbelloni LE, Beato L, Gouveia MA - Raquianestesia unilateral com bupivacaína hipobárica. Rev Bras Anestesiol, 2002; 52:542545.

11. Bromage PR - A comparison of the hydrochloride and carbon dioxide salts of lidocaine and prilocaine in epidural analgesia. Acta Anaesthesiol Scand, 1965;16:(Suppl):55-69.

12. Casati A, Fanelli G - Unilateral spinal anestesia. State of the art. Minerva Anestesiol, 2001;67:855-862.

13. Povey HM, Jacobsen J, Westergaard-Nielsen J - Subarachnoid analgesia with hyperbaric $0.5 \%$ bupivacaine: effect of $60-\mathrm{min}$ period of sitting. Acta Anaesthesiol Scand, 1989;33:295-297.

14. Esmaoglu A, Boyaci A, Ersoy O et al. - Unilateral spinal anaesthesia with hyperbaric bupivacaine. Acta Anaesthesiol Scand, 1998;42:1083-1087.

15. Kuusniemi KS, Pihlajamaki KK, Pitkanen MT - A low dose of plain or hyperbaric bupivacaína for unilateral spinal anesthesia. Reg Anesth Pain Med, 2000;25:605-610.

16. Cangiani LM - Determinação da densidade e da baricidade das misturas para anestesia subaracnóidea. Rev Bras Anestesiol, 2000;50:92-94.

17. Imbelloni LE, Beato L, Gouveia MA - Baixas doses de bupivacaína a $0,5 \%$ isobárica para raquianestesia unilateral. Rev Bras Anestesiol 2004;54:423-430. 
18. Tanasichuk MA, Schultz EA, Matthews JH et al. - Spinal hemianalgesia: an evaluation of method, its applicability, and influence on the incidence of hypotension. Anesthesiology, 1961; 22:74-85.

19. Ben-David B, Levin H, Solomon E et al. - Spinal bupivacaine in ambulatory surgery: the effect of saline dilution. Anesth Analg 1996;83:716-720.

20. Stienstra R, Green NM - Factors affecting the subarachnoid spread of local anesthetic solution. Reg Anesth, 1991;16:1-6.

21. Meyer J, Enk D, Penner M - Unilateral spinal anesthesia using low-flow injection through a 29-gauge Quincke needle. Anesth Analg, 1996;82:1188-1191.

22. Niemi L, Tuominen M, Pitkanen $M$ et al. - Effect of late posture change on the level of spinal anaesthesia with plain bupivacaine. $\mathrm{Br} J$ Anaesth, 1993;71:807-809.

23. Lotz SMN, Crosgnac M, Katayama M et al. - Anestesia subaracnóidea com bupivacaína 0,5\% hiperbárica: Influência do tempo de permanência em decúbito lateral sobre a dispersão cefálica. Rev Bras Anestesiol, 1992;42:257-264.

24. Kaya M, Oguz S, Aslan K et al. - A low-dose bupivacaine: a comparison of hyperbaric and hypobaric solutions for unilateral spinal anesthesia. Reg Anesth Pain Med, 2004;29:17-22.

25. Carpenter RL, Caplan RA, Brown DI et al. - Incidence and risk factors for side effects of spinal anesthesia. Anesthesiology, 1992;76:906-916.

26. Casati A, Fanelli G, Aldegheri G et al. - Frequency of hypotension during conventional or asymmetric hyperbaric spinal block. Reg Anesth Pain Med, 1999;24:214-219.

\section{RESUMEN}

Imbelloni LE, Beato L, Gouveia MA, Cordeiro JA — Baja Dosis de Bupivacaína Isobara, Hiperbara o Hipobara para Anestesia Raquidea Unilateral.

JUSTIFICATIVA Y OBJETIVOS: La anestesia raquidea unilateral puede presentar ventajas principalmente en pacientes en régimen ambulatorial. Baja dosis de la solución anestésica, velocidad lenta de la inyección espinal y la posición lateral facilitan la obtención de la distribución unilateral en la anestesia raquidea. Se compararon las soluciones isobara, hiperbara e hipobara de bupivacaína para obtener la anestesia raquidea unilateral en pacientes sometidos a intervenciones quirúrgicas ortopédicas en régimen ambulatorial.

MÉTODO: Ciento cincuenta pacientes fueron aleatoriamente separados en tres grupos para recibir $5 \mathrm{mg}$ de bupivacaína a 0,5\% isobara (Grupo Iso) o $5 \mathrm{mg}$ de bupivacaína a 0,5\% hiperbara (Grupo Hiper) o $5 \mathrm{mg}$ de bupivacaína a 0,15\% hipobara (Grupo Hipo). Las soluciones se administraron en el interespacio $L_{3}-L_{4}$ con el paciente en la posición lateral y permaneciendo en esa posición por 20 minutos. La anestesia sensitiva se evaluó por el test de la picada de la aguja. El bloqueo motor evaluado por la escala modificada de Bromage. Los dos bloqueos se compararon con el lado no operado y también entre sí.

RESULTADOS: Se notó una diferencia significativa entre el lado operado y no operado en todos los tres grupos a los 20 minutos, pero se obtuvo una mayor frecuencia de anestesia raquidea unilateral con las soluciones hiperbara e hipobara de bupivacaína. Se observó bloqueo sensitivo y motor en 14 pacientes del Grupo Iso, 38 pacientes en el Grupo Hiper y 40 pacientes en el Grupo Hipo. No ocurrieron alteraciones hemodinámicas en ningún paciente. No se observó cefalea postpunción ni síntomas neurológicos transitorios.

CONCLUSIONES: La anestesia raquidea con soluciones hipobara e hiperbara proporcionaron una mayor frecuencia de unilateralidad. Tras 20 minutos la solución isobara de bupivacaína se movió en el LCR resultando en apenas $28 \%$ de anestesia raquidea unilateral. 